\title{
STUDY ON BUILDING EXTRACTION FROM HIGH-RESOLUTION IMAGES USING MBI
}

\author{
Z. Ding *, X. Q. Wang, Y.L. Li, S.S. Zhang \\ Key Laboratory of Spatial Data Mining \& Information Sharing of Ministry of Education, National Eng.Research Center of satellite \\ spatial information technology, Fuzhou University, China,1512597818@qq.com
}

Commission III, WG III/1

KEY WORDS: Building Extraction, Morphological Building Index, Feature Extraction, High Resolution, Mathematical Morphology

\begin{abstract}
:
Building extraction from high resolution remote sensing images is a hot research topic in the field of photogrammetry and remote sensing. However, the diversity and complexity of buildings make building extraction methods still face challenges in terms of accuracy, efficiency, and so on. In this study, a new building extraction framework based on MBI and combined with image segmentation techniques, spectral constraint, shadow constraint, and shape constraint is proposed. In order to verify the proposed method, worldview2, GF-2, GF-1 remote sensing images covered Xiamen Software Park were used for building extraction experiments. Experimental results indicate that the proposed method improve the original MBI significantly, and the correct rate is over $86 \%$. Furthermore, the proposed framework reduces the false alarms by $42 \%$ on average compared to the performance of the original MBI.
\end{abstract}

\section{INTRUDUCTION}

Buildings are not only the most important place for human production and life, but also an important part of the urban environment. The precise detection of buildings is of great importance to urban planning and management, urban cadastral management, urban geo-database updating, disaster risk assessment and rescue (Huo et al.,2017). In recent years, with the increasing abundance of high-resolution remote sensing images and the gradual reduction of acquisition costs, the research on extracting buildings using high-resolution images has been rapidly developed. It gradually evolved from the single use of spatial information to the comprehensive utilization of spatial information, spectral information and structural information. However, due to the diversity of buildings, complex lighting conditions, shadow shading and other aspects, the method of Building Extraction from high-resolution images still faces many challenges in terms of extraction efficiency, degree of automation, and extraction accuracy of complex images. Therefore, the building extraction method with high efficiency, high precision and easy application not only has the methodological scientific significance but also has strong application value.

At present, there are mainly three types of methods for extracting buildings from high-resolution images. They are object-oriented method, method based on auxiliary features or information and building index method, respectively.

Object-oriented method uses image segmentation as the basis and object as the basic unit, avoiding the interference of the extra detailed information in the high-resolution images to the building extraction. It can make full use of the many features of the object to effectively extract the buildings, and has become the mainstream method of extracting buildings using high-resolution images (Johnson \&Xie,2013; Blaschke T,2010). However, this method is easily restricted by image segmentation. If the segmentation object can not accurately describe the shape, location and other features of the object, it will seriously affect the extraction accuracy. Due to the limitation of imaging angle, many features of real buildings can not be displayed in the image. Method based on auxiliary features or information introduces the support of auxiliary feature information, so as to further expand the development direction of building identification methods and improve the accuracy of identification. Based on the feature that buildings are significantly above the ground, buildings are often easily extracted by height thresholds based on DEM data (Tournaire et al.,2010) or Digital Surface Model (DSM) (Maruyama et al.,2011). Shadows can reflect the threedimensional information of objects (Izadi, al.,2012). Building shadows play a very important role in enhancing the accuracy and reliability of building detection.

Building index can be directly applied to the extraction of buildings, and does not require training and segmentation process, so that the extraction efficiency is greatly improved. Building indices include the Texture-derived Build-up presence(PanTex) Index (Pesaresi, al.,2008), discrimination by Ratio of Variance(DRV) Index (LHOMME, al.,2009), Morphological Building Index (MBI) ( Huang \& Zhang,2011), etc. One of the most widely used is MBI, it can extract building information from images without any training process, with better automation and extraction accuracy. However, MBI is subject to commission and omission errors. Bright soils, open areas and roads are mistakenly classified as buildings, as they are also brighter than neighborhoods and show similar spectral characteristics to buildings. In this study, a new building extraction framework based on MBI is designed to detect buildings in different resolution images. The proposed framework can efficiently remove these false alarms by combining of MBI and objectoriented object information extraction idea, and taking full account of the spectral, geometrical and contextual information of buildings to complete the building extraction.

\footnotetext{
* Zhe Ding, male, postgraduate, mainly engaged in remote sensing research on natural resources and the environment. Email: dingzhegis@qq.com
} 


\section{STUDY AREA AND DATASET}

The study area is located at Xiamen software park $\left(24^{\circ} 29^{\prime} 22\right.$ "N, $\left.118^{\circ} 10^{\prime} 39^{\prime \prime} \mathrm{E}\right)$ in the east of Xiamen island, Fujian province. It was basically completed by the end of 2006 . We selected three high-resolution remote-sensing images covering Xiamen software park to validate the performance of the proposed framework. The details of these datasets are listed in Table 1.
These images selected in this paper all adopt the Gram-Schmidt (GS) algorithm to fuse the panchromatic and multispectral images. The fusion method can better improve the spatial detail of the original image and has good fidelity to the spectral features of the image (Aiazzi, et al.,2007). Three fusion images are shown in Fig.1.

\begin{tabular}{ccccc}
\hline \multirow{2}{*}{ Satellite sensor } & $\begin{array}{c}\text { panchromatic / Multi-Spectral } \\
\text { Spatial resolution/(m) }\end{array}$ & $\begin{array}{c}\text { solar azimuth } \\
/\left({ }^{\circ}\right)\end{array}$ & $\begin{array}{c}\text { solar altitude } \\
/\left(^{\circ}\right)\end{array}$ & imaging time \\
\hline WorldView-2 & $0.5 / 2$ & 150.3999 & 52.5000 & $2014-10-16$ \\
GF-2 & $1 / 4$ & 155.1705 & 54.3653 & $2015-10-15$ \\
GF-1 & $2 / 8$ & 162.3680 & 48.1327 & $2017-02-06$ \\
\hline
\end{tabular}

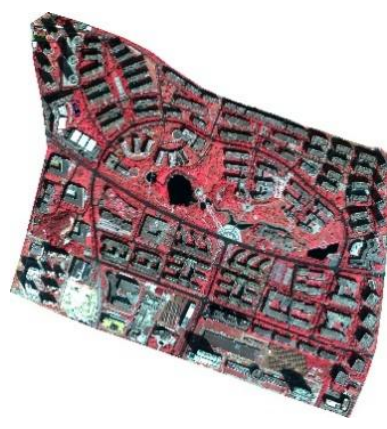

(a) WorldView-2

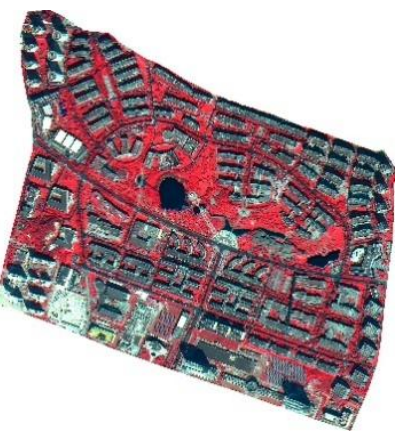

(b) GF-2

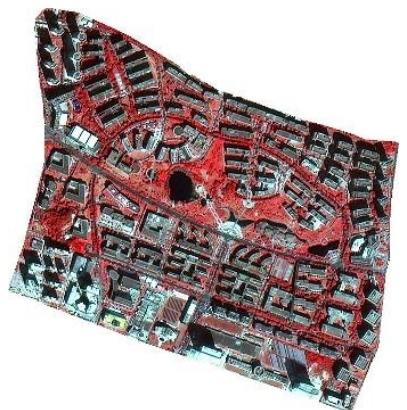

(c) GF-1

Figure 1. Three kinds of data fusion images of the test site

\section{METHODS}

Based on the MBI and the image segmentation technology, spectral constraints, shadow verification and shape constraints are applied to the building candidate regions satisfying the MBI threshold to improve the accuracy and automation of building extraction. Figure 2 shows the process flow of building extraction.

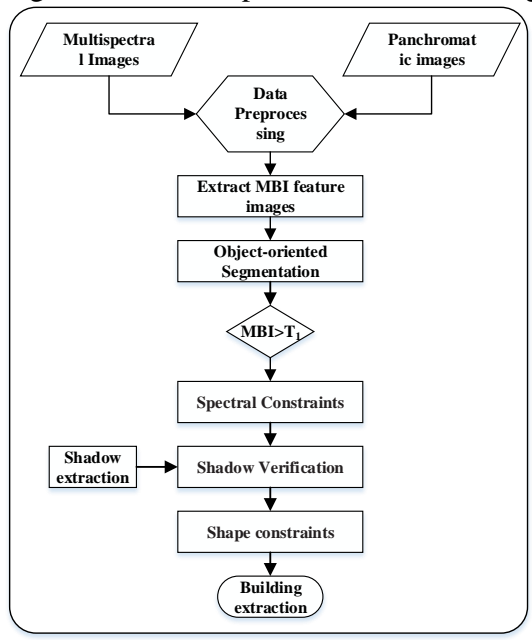

Figure 2. Flow chart of the proposed building extraction framework

\subsection{Morphological building index(MBI)}

MBI can be automatically calculated by the following steps: - Step 1) Construction of feature images: Based on the goal of restraining vegetation information and highlighting the building information, a feature image $\mathrm{b}(\mathrm{x})$ construction method using comprehensively the image red band and normalized vegetation index (NDVI) is proposed (Chen, al.,2016). Vegetation information is reflected by a highly acceptable NDVI, while building information is characterized by a red band that provides abundant surface information.

$$
\mathrm{b}(\mathrm{x})=\mathrm{b}_{\mathrm{red}} \times(1-\mathrm{NDVI})
$$

Where $b_{\text {red }}$ represent the reflectance values in the red channel.

- Step 2) Construction of MBI: The spectral-structural characteristics of buildings are represented using the DMP of the top-hat by reconstruction with a series of linear SE (Huang, al.,2017). The construction of MBI is based on the building structures have larger values in most directions of DMP histogram. Because the shadow around the building has low brightness value, they show high local contrast. Therefore, the buildings often refer to the big MBI value. Figure 3 shows the MBI feature image of GF-2.

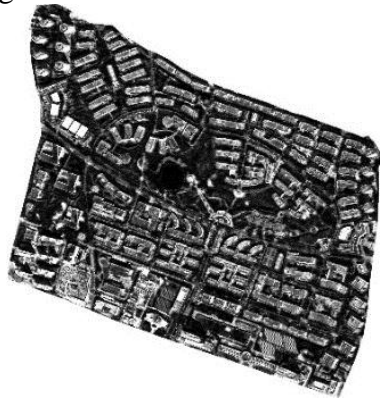

Figure3. MBI feature image of GF-2. 


\subsection{Building extraction process}

The proposed framework sequentially conducts spectral constraints (abbreviated as SPE), shadow verification (abbreviated as SHD), and shape constraints (abbreviated as SHA) after image segmentation.

\subsubsection{Spectral Constraints}

The hue component $(\mathrm{H})$, and the NDWI are used to refine the output of the original MBI. In the building extraction process, bright soil is the main source of misclassification and the color tone $(\mathrm{H})$ is introduced to reduce the erroneous classification of bare soil into buildings. NDWI is useful for improving building extraction accuracy and can be used for water removal, the rules are as follows:

IF NDWI(x) $\geq \mathrm{w}$, or $h_{\min }<\mathrm{H}(\mathrm{x})<h_{\max }$,

THEN $\mathrm{x}$ is identified as a non - building structure

where NDWI(.) is the NDWI feature value and $\mathrm{w}$ is the water index threshold. $\mathrm{H}($.) denote hue component $(\mathrm{H})$ feature values. hmin represents a fixed value of the green component and hmax represents a fixed value of the yellow component in the HSV space.

\subsubsection{Shadow Verification}

Shadow can be a constraint on building extraction to eliminate misclassifications such as soil, roads, parking lots, and open areas. Because the shadows of buildings show low brightness value on the remote sensing image, shadows can be extracted by setting the appropriate threshold in the near-infrared band of the remote sensing images. The resulting shadow feature image is expanded with linear structural elements in the opposite direction to the solar illumination angle. Shadow verification test whether the candidate buildings are adjacent to the shadow they cast. If there is an overlap, the detected building can be preserved; otherwise, the detected buildings are removed.

$$
\text { IF SPE }(x) \cup \operatorname{dilate}(S(x))=\emptyset \text {, }
$$

THEN $\mathrm{x}$ is identified as a non-building structure

where $\operatorname{SPE}(\mathrm{x})$ denotes the refined building detection result of pixel $\mathrm{x}$ after the spectral constraint, $\mathrm{S}($.$) is the shadow map, and$ dilate(.) represents the morphological dilation operator.

\subsubsection{Shape Constraints}

Shape features can be used as a constraint on building extraction. Based on the building map generated by the connected component analysis, the area is calculated by counting the total number of pixels of the object. The Geometrical index(GI) is used to characterize the shape of a building. Since buildings have greater rectangular fit and smaller length-width ratio than roads, they always have large GI feature values. The rule of SHA can be expressed as

$$
\text { IF Count }(\mathrm{x})<\mathrm{c}, \mathrm{OR} \text { GI }(\mathrm{x})<\mathrm{g}
$$

THEN $\mathrm{x}$ is identified as a non-building structure

where Count(.) is the number of pixels in an object and c represents its threshold. GI(.) denotes the Geometrical index and $\mathrm{g}$ is its threshold.

\subsection{Building extraction accuracy evaluation}

In order to evaluate the effectiveness of the proposed method, the following four building extraction indicators were used for accuracy evaluation in this paper. CT, MA and FA are correct rate, miss alarms and False Alarms. CT indicates that the accuracy of a real building can be detected. MA indicates the probability that a real building will be misjudged to be a nonbuilding during the extraction process. FA indicates the probability that a non-building misjudged as a building during the extraction process. OA is a comprehensive evaluation index which takes into account the accuracy and completeness of test results.

$$
\begin{gathered}
\mathrm{CT}=\frac{\mathrm{N}_{a}}{\mathrm{~N}_{a}+\mathrm{N}_{b}} \times 100 \% \\
\mathrm{MA}=\frac{\mathrm{N}_{C}}{\mathrm{~N}_{a}+\mathrm{N}_{C}} \times 100 \% \\
\mathrm{FA}=\frac{\mathrm{N}_{b}}{\mathrm{~N}_{a}+\mathrm{N}_{C}} \times 100 \% \\
\mathrm{OA}=\frac{\mathrm{N}_{\mathrm{a}}}{\mathrm{N}_{a}+\mathrm{N}_{b}+\mathrm{N}_{C}} \times 100 \%
\end{gathered}
$$

where $\mathrm{Na}$ denote the number of building pixels extracted from the experiment results; $\mathrm{N}_{\mathrm{b}}$ represent the number of building pixels that are wrongly extracted from the experimental results; and $\mathrm{Nc}$ denote the number of building pixels that are not extracted in the experimental results.

\section{RESULTS AND DISCUSSION}

MBI feature images were obtained by constructing linear structural elements from 4 directions $\left(0^{\circ}, 45^{\circ}, 90^{\circ}, 135^{\circ}\right)$ and 50 scales. The segmentation parameters of WorldView-2, GF-2 and GF-1 are 80,60, and 25 respectively, the shape parameters of the three images are all 0.6 , the compactness parameters of the three images are all 0.4 , by means of repeated segmentation experiment and comparison of the effect of different parameters. The building and shadow detection results are shown in Fig. 4, where the red and wathet pixels represent the detected buildings and shadow, respectively. Furthermore, It can be seen from Figure 4 that all the buildings and shadows of the three images are well extracted by selecting the appropriate segmentation scale and parameters.

The MBI method is used to compare, in the GF-1 test image (Fig. 5). The quantitative accuracies are listed in Table 2 . As can be seen from Fig. 5(a) and Fig. 5 (b), extraction results exist part of the buildings are not divided and serious irregular shape, and a large number of roads and bare land are divided into buildings by using the original MBI method. However, the method adopted in this paper can well reduce the number of buildings that are misclassified into other ground object. The extracted buildings are more complete and the outline is more regular.

According to Table 2, it is apparent that the proposed framework is superior to the original MBI in both completeness and correctness. Compared to the MBI method, the decrease of MA, FA are $19.3 \%, 42.2 \%$ in the WorldView-2 image, $19.4 \%, 43.1 \%$ in GF-2 image, and $22.7 \%, 43.4 \%$ in GF-1 image, respectively. Meanwhile, the increase of CT, OA are both more than $30 \%$ in all three test images. The improvement of CT, OA and the decrease of MA, FA indicates that a great deal of false alarms were removed by the use of the proposed framework. Whether using the proposed building extraction method or MBI method, the OA of WorldView-2 is the highest, followed by GF-2 and lowest is GF-1. Meanwhile, the MA of GF-1 is the highest, followed by GF-2 and WorldView-2 is lowest. 


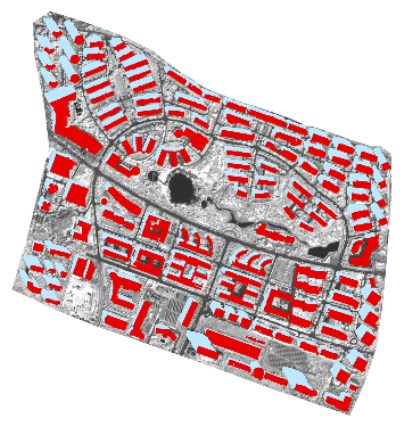

(a) WorldView-2

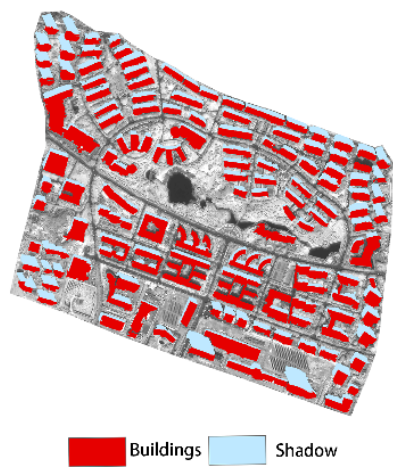

(b) GF-2

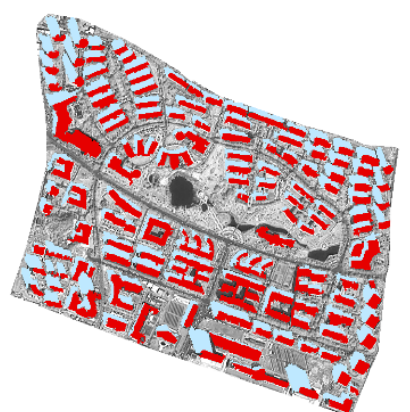

(c) GF-1

Figure 4. Building and shadow extraction results for three test images

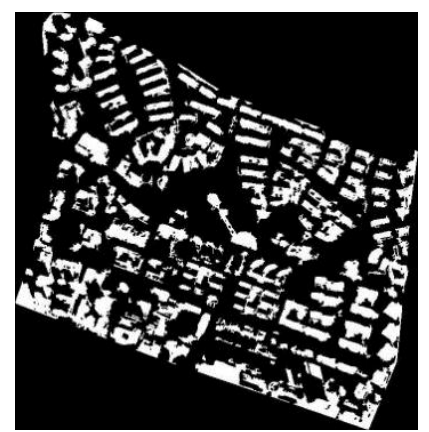

(a)MBI method

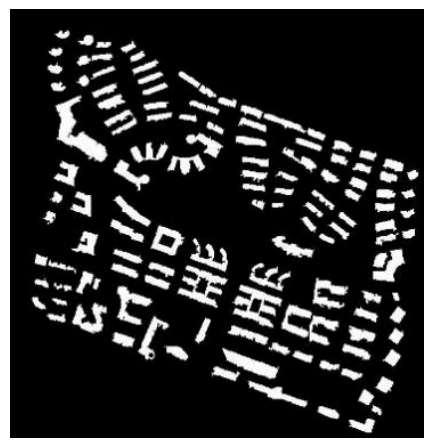

(b) the proposed framework

Fig.5 The result of building extraction from GF-1

\begin{tabular}{cccccc}
\hline Method & Satellite sensor & CT(\%) & MA(\%) & FA(\%) & OA(\%) \\
\hline \multirow{3}{*}{ The Proposed } & WorldView-2 & 92.8 & 10.9 & 6.9 & 83.4 \\
framework & GF-2 & 86.7 & 11.4 & 13.6 & 78.0 \\
& GF-1 & 89.8 & 20.5 & 9.0 & 72.9 \\
MBI method & WorldView-2 & 58.4 & 30.2 & 49.2 & 46.4 \\
& GF-2 & 55.0 & 30.8 & 56.8 & 44.4 \\
& GF-1 & 52.1 & 43.3 & 52.4 & 37.2 \\
\hline
\end{tabular}

Table 2 Accuracy assessment of building extraction result

\section{CONCLUTIONS}

In this paper, a new building extraction framework has been proposed, aiming at solving the shortcomings of MBI method. The proposed framework combines MBI with image segmentation technology, and introduces spectral constraints, shadow Verification, and shape constraints sequentially. WorldView-2, GF-2, GF-1 were used to validate the proposed framework, and the results were promising and satisfactory, the CT of all the three test images were higher than $86 \%$. Meanwhile, combining the evaluation indexes of four buildings' extraction precision, overall, as the spatial resolution of remote sensing images decreases, the accuracy of building extraction gradually decreases.

Due to the fact that the Geometrical index, area in the shape constraints are experimentally set. The rapid setting of the threshold has always been a difficult task for thematic information extraction. It needs to be improved afterwards. Future research includes further enhance the automation of the proposed process. At the same time, to further enhance the degree of automation of the building extraction framework is the focus of future research.

\section{ACKNOWLEDGEMENTS}

This work was supported by the Central Government Guides Local Development Special Fund under Grant No. 2017L3012.

\section{REFERENCES}

Aiazzi, B., Baronti, S., Selva, M., Alparone , L., 2007. Enhanced Gram-Schmidt Spectral Sharpening Based on Multivariate Regression of MS and Pan Data[C]// IEEE International Conference on Geoscience and Remote Sensing Symposium. IEEE, pp. 3806-3809.

Blaschke, T., 2010. Object based image analysis for remote sensing $[\mathrm{J}]$. Isprs Journal of Photogrammetry \& Remote Sensing, 65(1), pp. 2-16.

Chen, X., Zhuo, L., Tao, H.-Y., 2016. Study on Building Extraction from High Spatial Resolution Images Using MMBI[J]. Remote Sensing Technology and Application, 31(5), pp. 930-938. 
Huo, S.-F., Gu, X.-F., Zhan, Y.L.,2017. A method for estimating building heights based on ZY-3 satellite forward image [J],Science of surveying and Mapping, 42(2), pp. 147-153.

Huang X., Yuan, W.-L., LI, J.-Y., Zhang, L.-P, 2017. A New Building Extraction Postprocessing Framework for High-SpatialResolution Remote-Sensing Imagery [J]. IEEE Journal of Selected Topics in Applied Earth Observations and Remote Sensing, 10(2), pp. 654-668.

Huang, X., Zhang, L., 2011. A Multidirectional and Multiscale Morphological Index for Automatic Building Extraction from Multispectral GeoEye-1 Imagery[J]. Photogrammetric Engineering \& Remote Sensing, 77(7), pp. 721-732.

Izadi, M., Saeedi, P.,2012. Three-Dimensional Polygonal Building Model Estimation From Single Satellite Images[J]. IEEE Transactions on Geoscience \& Remote Sensing, 50(6), pp. 2254-2272.

Johnson, B., Xie, Z., 2013. Classifying a high resolution image of an urban area using super-object information[J]. Isprs Journal of Photogrammetry \& Remote Sensing, 83(3), pp. 40-49.

LHOMME, S., HE, D. C., WEBER, C., Morin, D., 2009. A new approach to building identification from very high spatialresolution images [J]. International Journal of Remote Sensing, 30(5), pp. 1341-1354.

Maruyama, Y., Tashiro, A., 2011. Yamazaki F. Use of Digital Surface Model Constructed from Digital Aerial Images to Detect Collapsed Buildings during Earthquake[J]. Procedia Engineering, 14(2259), pp. 552-558.

Pesaresi, M., GERHARDINGER, A., KAYITAKIRE, F., 2009. A Robust Built-Up Area Presence Index by Anisotropic Rotation-Invariant Textural Measure [J]. IEEE Journal of Selected Topics in Applied Earth Observations \& Remote Sensing, 1(3), pp. 180-192.

Tournaire, O., Brédif, M., Boldo, D., Durupt, M., 2010. An efficient stochastic approach for building footprint extraction from digital elevation models[J]. Isprs Journal of Photogrammetry \& Remote Sensing, 65(4), pp. 317-327. 